\title{
Why are new graduate nurses leaving the profession in their first year of practice and how does this impact on ED nurse staffing? A rapid review of current literature and recommended reading
}

\author{
By Michael Sandler, RN, BSN, CNCC(C)
}

\section{Key messages}

- Job demands such as heavy workload, high job stress, poor nursing-medicine relationships, lack of leadership or supervision and bullying lead to burnout and predict intent to leave.

- Newly graduated nurses perceive significant stress in their first year of practice regardless of context and background factors, such as hospital level (referral, rural, etc.) or the amount of experience they gained in their last clinical practicum.

- New graduate nurses' work behaviour is a complex process, influenced by their attitudes toward their work, personal characteristics, job opportunities, and workplace attributes. Several characteristics are significant in predicting satisfaction, organizational commitment and intent to leave over and above work attitudes.

- New graduate nurses' intent to leave correlates to their self-image and understanding on what it means to be a nurse.

- New graduate nurses' intent to leave decreases as they reach their second year of practice.

- Adequate support resources significantly reduced new graduate nurses' intent to leave.

\section{Definitions}

A new graduate nurse is defined as a nurse who has graduated from an accredited nursing school in the last two years and is licensed.

\section{Intended audience}

This review is most relevant for hospital administrators, emergency department unit managers and staff

\section{Method of research collection}

A literature search of CINAHL and MEDLINE was conducted after a brief exploration of the current literature. CINAHL was searched using the terms: new graduate nurses, personal retention, job satisfaction, and work environment. MEDLINE was searched, using the terms job satisfaction, health personnel, retention psychology and new graduate nurs*.

A total of 139 articles were reviewed for appropriateness. One hundred and thirty-two were discarded. Six articles were used for this review. A subsequent second search was conducted with ED nursing as a modifier and a further 12 articles were reviewed to provide recommendations relevant to the emergency department (ED) nursing environment.

\section{Limitations of the research}

1. This summary includes solutions targeted to the issues of new graduate ED nurse retention.

2. Generally, surveys are subject to selection bias.

3. Most of the research included in this review was conducted in North America, leading to a geographical bias.

4. Some of the foundational research on this was conducted in the 1970s and not included in this review.

\section{Background}

The attrition rate of new graduate nurses remains higher than in other areas of the nursing workforce (Kramer, Brewer \& Maguire, 2013). This is concerning given the significant resources that are needed to transition new nurses into practice. Further, the nursing workforce is not currently able to absorb the added human resource losses. Previous research has established that up to $18 \%$ to $30 \%$ of new graduates will leave their current position for a different practice environment or the profession all together in the first year and up to $37 \%-57 \%$ will leave in their second year of practice. High turnover rates can have a lasting negative impact on staff morale, work productivity, and even patient outcomes (Chandler, 2012).

\section{Evidence}

The most common causes of new graduate nurses increasing "intent to leave" included interpersonal violence, workplace stress, feelings of incompetence and low confidence related to practice, unit workload and interdisciplinary relationships. An important finding that emerged as a primary reason for new graduates leaving practice altogether was interpersonal violence within nursing and then between nursing and other healthcare professionals, such as doctors.

1. Multiple dimensions of graduate nurse self-concepts rise significantly in the second half of the graduate year and nurses who report a strong self-concept correlate with a strong predictor of graduate nurse retention.

2. Poor work environments, poor nursing supervision, and difficult physician relations along with high workload, inadequate staffing, and time pressures lead to stress that is cited as a primary reason to leave.

3. Job demands predicted burnout and subsequently poor mental health. Job resources predicted work engagement and lower turnover intentions. Burnout was also a significant predictor of turnover intent. 
4. Newly licensed registered nurses' work behaviour is a complex process, influenced by their attitudes toward their work, personal characteristics, job opportunities, and workplace attributes. Several characteristics are significant in predicting satisfaction (ethnicity, gender) and organizational commitment (patient load, mandatory overtime, shift, and unit type) and intent to stay (income, age) over and above work attitudes.

5. The level of anxiety and stress experienced by new graduate nurses is extraordinary. In the acute care clinical environment, graduate nurses' responsibility is overwhelming. Stress in the clinical practice environment is one of the many contributing factors that influences new graduate nurses' intention to quit in their first 12 months of nursing practice.

6. Newly graduated nurses perceived a lot of stress and nearly one-third of them intended to quit during their first three months of clinical practice. Background factors such as hospital level (referral, rural, etc.) and the nurses' experience in their last clinical practicum are the best predictors of the intention to quit as new grads.

\section{Bottom line}

Each setting will have a different collection of predictors of intent to leave that intersect to affect the new graduate negatively. Resources should be placed strategically to enhance support and reduce these predictors. Targeted solutions based on best practice and evidence should be explored to support new graduate nurses.

\section{Targeted ED findings}

1. Research suggests that the total cost to mentor three new graduate nurses to the ED using an extended residency is approximately $\$ 90,000$, providing a savings of more than $\$ 100,000$ compared to the cost of $\$ 192,000$ to recruit three new staff.
2. New graduate nurses who participated in a targeted ED residency program suggested that six months was an appropriate length of program to meet objectives and develop the required skills to provide high-quality patient care.

3. A dedicated supernumerary mentor for new graduate ED nurses led to better patient care outcomes, increased staff morale, better inter-professional relationships, and more overall unit efficiency including increased patient flow through the department.

4. To be successful, new graduate nurses in the ED require access to strong clinical and administrative leadership. Ensure accessible leadership, management and governance structures are in place. Create smaller teams, as part of the larger overall structure to foster increased support for new graduate emergency nursing practice (Evans, Hughes, \& Ward, 2017; Turner \& Goudreau, 2011; Betts, 2003).

\section{About the author}

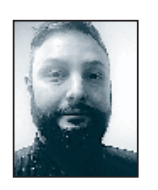

I (Michael Sandler) am a passionate nurse educator with an interest in promoting, transport and rural and remote nursing, addressing and/or expanding scope of practice issues, and improving the care received by patients throughout B.C. I relocated to the beautiful B.C. interior to take on the role of Clinical Practice Educator (CPE) for the High Acuity Response Team (HART), which is an innovative practice that addresses the provision of mobile intensive care to rural hospitals that do not have critical care services in an effort to stabilize and sustain patient care and, when necessary, transport patients to a higher level of care. The practice was recognized as a Promising Practice by the Health Council of Canada using the Health Innovation Portal Evaluation Framework. I also have the pleasure of chairing Association of Registered Nurses of British Columbia's (ARNBC) Rural and Remote policy table, a joint effort by the B.C. Ministry of Health and $A R N B C$ to bring a nursing focus to rural and remote healthcare issues, including improving nursing services to rural and remote communities. In my personal time, I am completing graduate studies in nursing at University of British Columbia-Okanagan.

\section{REFERENCES}

Betts, L. (2003). Mentorship. an emergency department recruitment strategy. The Canadian Nurse, 99(9), 28.

Chandler, G.E. (2012). Succeeding in the first year of practice: Heed the wisdom of novice nurses. Journal for Nurses in Staff Development, 28(3), 103-107. doi:10.1097/NND.0b013e31825514ee

Evans, C., Hughes, C., \& Ward, K. (2017). Improving staff recruitment and retention in the emergency department. Nursing Times' 113(4), 18-21.
Kramer, M., Brewer, B.B., \& Maguire, P. (2013). Impact of healthy work environments on new graduate nurses' environmental reality shock. Western Journal of Nursing Research, 35(3), 348-383. doi: $10.1177 / 0193945911403939$

Turner, N., \& Goudreau, J. (2011). From student to emergency nurse: A qualitative study of an educational support seminar. Journal for Nurses in Staff Development, 27(5), 210-215. doi:10.1097/ NND.0b013e31822e0655

\section{RECOMMENDED ARTICLES}

Cowin, L. S., \& Hengstberger-Sims, C. (2006). New graduate nurse self-concept and retention: A longitudinal survey. International Journal of Nursing Studies, 43(1), 59-70. doi:10.1016/j. ijnurstu.2005.03.004

Kovner, C.T., Brewer, C.S., Greene, W., \& Fairchild, S. (2009). New registered nurses' intent to stay at their jobs. Nursing Economic, 27(2), 81-98.

McCalla-Graham, J.A., \& De Gagne, J.C. (2015). The lived experience of new graduate nurses working in an acute care setting. Journal of Continuing Education in Nursing, 46(3), 122. doi:10.3928/00220124-20150220-17
Scott, E.S., Keehner Engelke, M., \& Swanson, M. (2008). New graduate nurse transitioning: Necessary or nice? Applied Nursing Research, 21(2), 75-83. doi:10.1016/j.apnr.2006.12.002

Spence Laschinger, H.K., Grau, A.L., Finegan, J., \& Wilk, P. (2012). Predictors of new graduate nurses' workplace well-being: Testing the job demands-resources model. Health Care Management Review, 37(2), 175-186

Yeh, M., \& Yu, S. (2009). Job stress and intention to quit in newlygraduated nurses during the first three months of work in Taiwan. Journal of Clinical Nursing, 18(24), 3450. doi:10.111 1/j.1365-2702.2009.02941. 